\title{
Minimally Invasive Approach for Rhinoplasty
}

\author{
S. Cohen \\ Plastic and Aesthetic Surgery, Private Clinic, Ramat Gan \\ Israel
}

\section{Introduction}

The nose, the most prominent aesthetic feature in the facial profile, is a three-dimensional, intricate trapezoid solid, encompassing the external bony and cartilaginous vault with an overlying skin cover and internal cavities. Through their intricate structural interdependency, these topographic features contribute both to form and function. Given its central location in the midface, the nose interrelates with the adjacent structures through juxtaposition, ultimately giving rise to the overall size, shape, and aesthetics of the nose.

Ideal facial and nasal forms have been depicted from ancient Egyptian hieroglyphics to the Renaissance era. These cannons dictated what is most desirable and possibly achievable. Correction of aesthetic nasal deformities date back to India in 600 BC by Sushruta Samhita. Since then, there has been a long evolution of techniques. Different surgical approaches have been advocated, each with its inherent advantages and liabilities. Optimal rhinosculpturing outcomes are not achieved merely as a consequence of the access route but rather rely on a precise execution of a technique that addresses the deformity.

Science and art are inseparable in rhinoplasty. Gratifying results performed by surgical intervention or minimally invasive procedures require systematic analysis of the anatomic variables, adherence to structured strategy, artistic perception, and precise manipulation. It is a well known tenet to all surgical endeavors that a sound knowledge of anatomy is requisite and the reader is advised to refer to a comprehensive review of Surgical anatomy of the nose, by O'Neal, et al. 1996 (1).

\section{Nasal aesthetics}

Balance, harmony and symmetry are essential elements of beauty; thus, a thorough nasal and facial analysis in frontal, lateral, and basal views is of paramount importance in achieving state-of-the-art results in rhinoplasty.

Although a complete description of nasal analysis is beyond the scope of this article, certain fundamental considerations merit discussion.

In the frontal view, assessment of balance is achieved by dividing the face into horizontal thirds (trichion to glabella, glabella to subnasale, and subnasale to menton), and vertical fifths. The nose should represent one-third of the length of the face and one-fifth, the width (Figure 1). The ideal shape of the nose is outlined by two slightly curved divergent lines extending from the medial brows to the tip-defining points. The width of the alar base is equal to the intercanthal distance (Figure 1). The width of the nasal base comprises approximately $70 \%$ to $80 \%$ of the alar base. 


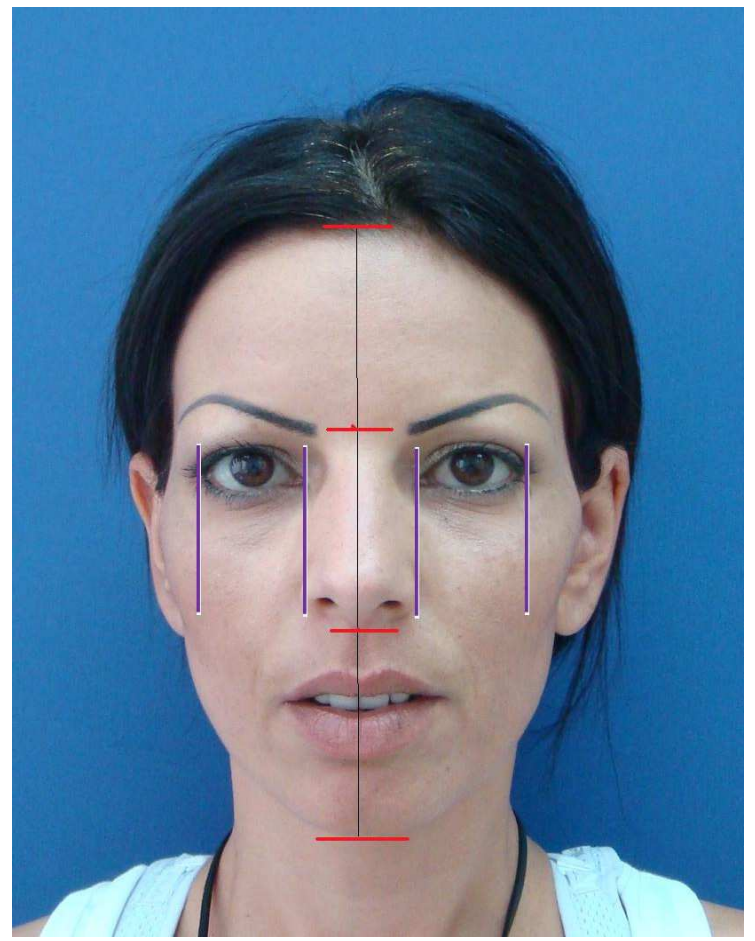

Fig. 1. The face is divided into thirds by horizontal lines drawn from the hair line, glabella, subnasale and menton. The nose should represent one-third of the length of the face and one-fifth of its width. Alar base is equal to the intercanthal distance

In the lateral view, important parameters include nasal length, tip rotation, tip projection and dorsal contour. Nasal length (or dorsal length) is determined by the vertical distance from the nasion (root of the nose overlying the nasofrontal suture) to the tip-defining point. Tip rotation is equivalent to the nasolabial angle, which measures the rotation of the nasal base from the upper lip. In women, it is $95^{\circ}$ to $105^{\circ}$. In men, it is $90^{\circ}$ to $95^{\circ}$ (Figure 2). Nasal tip projection is commonly assessed by Goode's method (2). Goode defines ideal nasal projection (measured from the alar crease to the tip-defining point) as 0.55 to 0.60 in relation to the dorsal length. Assessment of the dorsal contour should identify any concavity, convexity or irregularity. In women, the aesthetic nasal dorsum lies approximately $2 \mathrm{~mm}$ behind and parallel to a line from the nasofrontal angle to the tip, with a slight supratip break offsetting the nasal tip from the dorsum. In men, the dorsum should be slightly higher and a subtle convexity is typical.

The relationship of the ala and columella is likewise assessed on profile. Acceptable columellar show is between $2 \mathrm{~mm}$ to $4 \mathrm{~mm}$.

On profile view, one should be acquainted with the Powell and Humphrey's aesthetic angles applied in facial analysis (2). Of these, nasofrontal and nasofacial angles should be carefully assessed. As an angle interrelates two juxtaposed lines, changing the inclination of one line will consequently alter the perceived overall proportions, and in particular, the apparent nasal length. 
The nasofrontal angle (the angle formed between the forehead inclination and the nasal dorsum) is $130 \pm 7$ degrees in men and $134 \pm 7$ degrees in women (Figure 3). A deep nasofrontal angle contributes to the illusion of a short nose (and apparent overprojection), and a shallow nasofrontal angle adds apparent length to the nose. The nasofacial angle refers to the inclination between the nasal dorsum and the frontal plane (defined as a line from the nasion to the pogonion, the most prominent anterior projection of the chin). It is $36^{0}$ in men and $34^{0}$ in women.

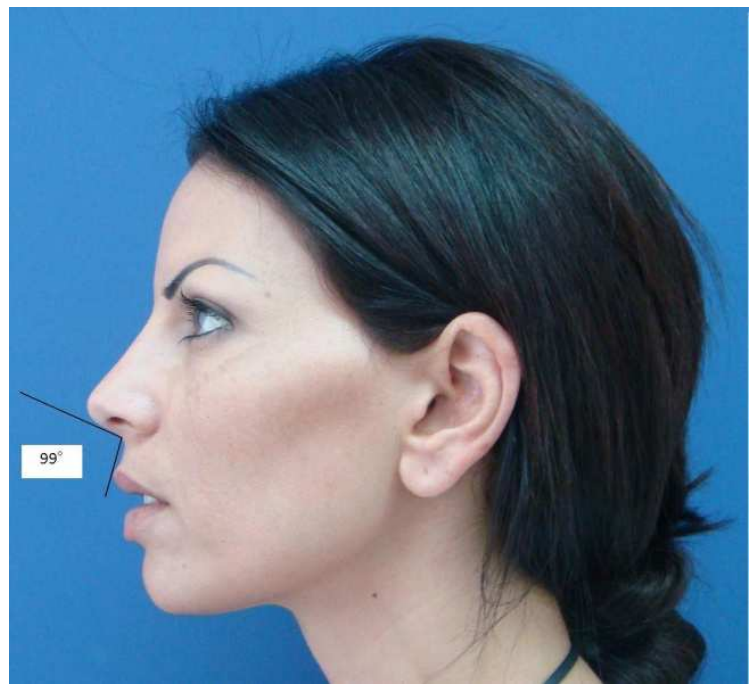

Fig. 2. Tip rotation is determined by the nasolabial angle. In women, it is $95-105^{0}$; in men, it is $90-95^{0}$

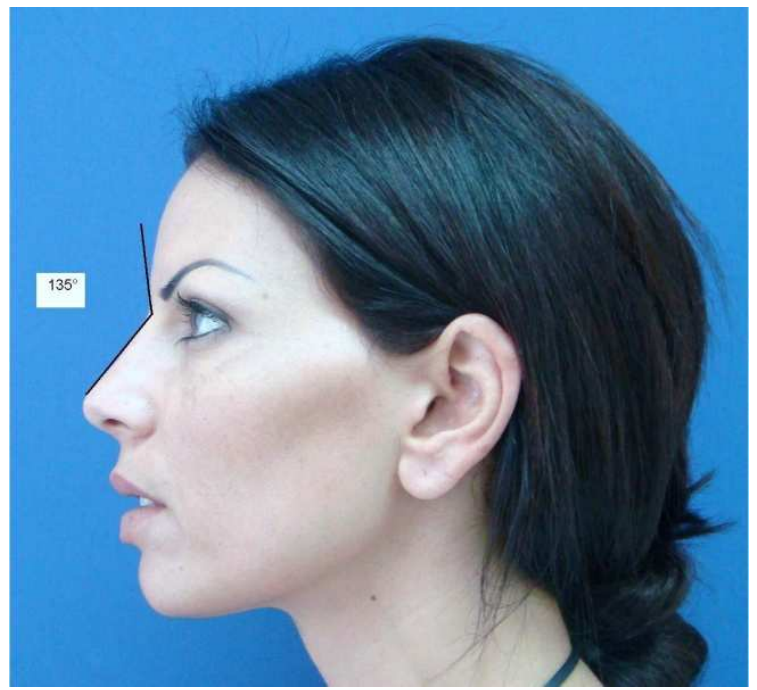

Fig. 3. Nasofrontal angle in women is $134 \pm 70$; in men, it is $130 \pm 70$ 
On base view, attention should be paid to symmetry, triangularity, columella-to-labial ratio, and width of the alar base. The nasal base should appear as an isosceles triangle with the upper third representing the tip lobule and the lower two-thirds corresponding to the columella.

As facial structures interrelate through juxtaposition, ultimately altered proportions of one part affect the perceived aesthetic appearance of adjacent parts. Nasal aesthetic appearance is likewise influenced by interrelated structures of the chin, forehead and premaxilla, and these should be individually analyzed.

Chin projection is assessed by a perpendicular line drawn between the Frankfurt horizontal line (FHL, a line connecting the superior aspect of the tragus to the infraorbital rim) and nasion. This perpendicular line should intersect the pogonion at $0 \pm 2 \mathrm{~mm}$. Alternatively, a perpendicular line from FHL through subnasale should intersect the pogonion $3 \pm 3 \mathrm{~mm}$ posterior to the line. An underprojected chin may lead to the perception of an overprojected nose and vice versa. A flat forehead gives the illusion of increased nose length (3).

As the premaxilla and the pyriform aperture serve as scaffolding for the nasal pyramid, anterior or posterior displacement of these anatomic landmarks may give the illusion of overprojection or retruded nasal profile, respectively. Deficiency of the premaxilla may present as a congenital deformity. Maxilla alveolar hypoplasia along with midfacial retrusion may be congenital or arise as part of the normal aging process (4). Loss of the underlying nasal support imparts an impression of a relative lengthening and retrusion to the aging nose (4)

Different aesthetic facial proportions exist among different faces and ethnicities. Nevertheless, similar concepts of beauty exist among people of various cultural backgrounds in cross-cultural studies (5). Accepted cultural standards and essential elements of beauty are the ultimate goals regardless of the patient's ethnic descent or race.

\section{Minimally invasive techniques in rhinoplasty}

During the last two decades, minimally invasive facial aesthetic procedures have gained widespread popularity. According to the American Society for Aesthetic Plastic Surgery (ASAPS), the annual number of nonsurgical cosmetic procedures increased by $228 \%$ from 1997 to 2010, and in 2010, nonsurgical procedures accounted for $83 \%$ of the aesthetic procedures performed overall (6).

While botulinum toxin injection tops the ASAPS's annual list as the most commonly performed cosmetic procedure since 2000, soft tissue augmentation procedures accounted for more than $15 \%$ of the procedures performed in 2010. Breakdown by filler type of soft tissue augmentation procedures reported by the ASAPS in 2010 indicated that calcium hydroxylapatite (CaHA) is the second most frequently used filler following hyaluronic acid (HA) filler derivatives.

Purported advantages over traditional surgeries include lack of scars, minimal down-time, less pain, and lower cost. The nose is amenable to a variety of minimally invasive techniques.

\subsection{Indications}

Minimally invasive nonsurgical techniques in rhinoplasty allow for gratifying results in a variety of indications, including augmentation type primary rhinoplasty(rhinosculpturing), correction of post-rhinoplasty contour defects, treating the aging nose, dealing with the ethnic nose and ameliorating/ reversing selected functional nasal impairments. 


\section{Augmentation type rhinosculpture}

Augmentation type, minimally invasive techniques might be utilized to correct both aesthetic and functional nasal deformities (i.e., internal valve collapse). Architectural deficiencies or imperfections necessitating augmentation type procedures can be systematically categorized by dividing the nose and nasal skeleton into thirds:

1. Upper-third - low radix (root of the nose), widened bony pyramid, bony contour irregularities.

2. Middle-third - low dorsum, dorsal contour irregularities, saddle nose deformity.

3. Lower-third - inadequate tip projection, underrotated tip, retracted columella.

\section{Upper-third}

Low radix. The radix corresponds to the nasofrontal groove at a level approximately between the supratarsal fold and the upper lash margin on straight gaze, and approximately 9-14 $\mathrm{mm}$ anterior to the corneal projection. When the radix begins lower than the upper lash margin, nasal length is shorter and the nasal base size appears to be larger. By augmenting a low radix, the nasofrontal angle is displaced cephalically, increasing the distance from nasion to the tip-defining point. This creates the perception of a larger appearing nose, improves the apparent overprojected tip, and gives rise to a more balanced and proportional aesthetic appearance.

Widened bony pyramid. The width of the bony vault should be analyzed both at the bony base and at the dorsal ridge. Ideal width varies from individual to individual and is influenced by the overall facial width, nasal length and projection, and by skin thickness. Apparent widening of the bony pyramid might be secondary to loss of height and light reflex from the nasal dorsum. In the case of a widened bony pyramid, augmentation of the central bony pyramid may effectively narrow the perceived width.

Bony contour irregularities. These irregularities can be de-emphasized and unsightly depressions can be eliminated or reduced.

\section{Middle-third}

Low dorsum. Dorsal height is assessed on profile view. Low dorsum refers to a dorsum that lies more than $2 \mathrm{~mm}$ behind and parallel to a line from the nasofrontal angle to the tipdefining point, and presents primarily as a congenital deformity, a consequence of previous trauma or as a common characteristic of the ethnic nose, such as the platyrrhine and the African American nose. Augmentation of a low dorsum is performed by using a dorsal graft or via a minimally invasive technique.

Saddle nose deformity. This deformity refers to an abnormally concave nasal dorsum in profile. Predisposing factors include previous trauma, rhinoplastic surgery (overresection of the septum, excessive dorsal hump resection), infectious disease (i.e., syphilis, leprosy), inflammatory disease (i.e., Wegener granulomatosis), neoplastic causes and platyrrhine nasal structure. Associated nasal defects commonly seen include cephalic tip rotation, middle vault collapse, and loss of tip support. In appropriately selected cases, correction for saddle deformity can be achieved by nasal dorsum augmentation alone, whereas more severe deformities mandate structural realignment with conventional approaches.

\section{Lower-third}

Inadequate tip projection. Tip projection is conventionally assessed by measuring the distance of the tip-defining point from a facial parameter (i.e., nasion, alar crease) $(7,8)$. 
However, in some cases, the nasal bases are large, yet the tip cartilages have poor projection. An alternative functional definition is proposed by Constantian (9). A tip with inadequate projection is defined as any tip that does not project to the level of the septal angle (identified in the supratip as the edge of the dorsal septal angle). An underprojected tip generates the illusion of a dorsal pseudo-hump in the supratip region, secondary to lack of support and discontinuity in the supratip-lobule region. Augmentation of the nasal tip and supratip lobule region can affectively support and project the tip and disguise discontinuity in the caudal dorsum.

Underrotated tip. As alluded to previously, the perceived degree of tip rotation is defined by the nasolabial angle. Desired tip rotation is influenced by gender and by the patient's height (inversely proportional). In women, it is 95 to 105 degrees, whereas a more acute angle of approximately 90 degrees is considered aesthetic in men. Cephalic or caudal positioning of the tip leads to corresponding change in the nasal length, tip rotation and columellar inclination. An overly obtuse nasolabial angle makes the nose appear short, whereas the converse adds apparent length. By augmenting a ptotic tip, the tip-defining point is displaced cephalically so the distance between nasion to the tip-defining point is reduced, thus making the nose appear shorter and aesthetically projected. Augmentation of the anterior nasal spine of the maxillary bone located at the central part of the nasal base opens the nasolabial angle and rotates the tip cephalically.

\section{Post-rhinoplasty deformities}

Postoperative rhinoplasty complications range from $8 \%$ to $15 \%$ (10) and result primarily from failure to maintain adequate cartilaginous and bony structural support. Aesthetic deformities often have functional implications and reflect the interdependency of form and function.

Detailed systematic analysis of each of the structural and functional anatomic variables is of utmost importance to determine the correct diagnosis and to properly select a treatment plan. Postoperative complications that result from overresection/overcorrection following overzealous surgery are often amenable to augmentation techniques and will be presented in accordance to the nasal thirds:

1. Upper-third (nasion to rhinion) - low radix, low dorsum, dorsal irregularities, skeletal deformities.

2. Middle-third - inverted V deformity, supratip deformity, saddle nose deformity.

3. Lower-third - external valve incompetence, loss of tip support.

\section{Upper-third}

Dorsal irregularities result from unsmoothed residual bony or cartilaginous ("mid-dorsal notch") fragments following hump removal. Poorly performed osteotomies result in palpable skeletal deformities. These include "open roof" deformity, "step off' deformities and Rocker deformity. Rocker deformity occurs when medial osteotomy is taken too high into the thick frontal bone. Consequently, the superior aspect of the osteotomized nasal bone projects or "rocks" laterally. By augmentation of these untoward postrhinoplasty sequelae, these deformities are de-emphasized or even eliminated. Likewise, an overresected dorsum or low radix, otherwise necessitating dorsal or radix grafts, can be successfully augmented using minimally invasive procedures. 


\section{Middle-third}

Inverted $\mathbf{V}$ deformity refers to inferomedial collapse of the upper lateral cartilage (ULC) consequent to inadequate support of the ULC following overresection of the cartilaginous roof during hump removal. At its caudal end, the ULC ideally forms an angle of 10 to 15 degrees with the septum near the anterior-septal angle. This region is defined as the internal nasal valve and requires patency for normal airway.

When the middle vault collapses towards the anterior septal edge, internal nasal valve collapse ensues, resulting in nasal airway obstruction and inverted V deformity.

Alignment of the internal valve area can improve the nasal airway and disguise the accompanying aesthetic deformity, thus targeting both form and function. Traditionally, correction of internal valve incompetence is accomplished by placing spreader grafts, either unilaterally or bilaterally, that serve as spacer grafts between the dorsal septum and upper lateral cartilage during rhinoplasty.

Supratip deformity (Polly beak) refers to postoperative fullness of the supratip and a blunt tip lobular poorly differentiated from the dorsum. Inadequate tip support and over-resection of the bony hump or cartilage [e.g., dorsal septum, dome or lower lateral cartilage (LLC)] are etiologies that are amenable to augmentation by minimally invasive techniques, with injection to appropriate areas simulating the effect of a columellar strut and dorsal grafts.

\section{Lower-third}

External valve incompetence. The external nasal valve refers to the area delineated by the cutaneous and skeletal support of the mobile alar wall. Overresection of the lateral crus can lead to collapse of the external valve with negative pressure of respiration, and consequent nasal airway obstruction. Alar retraction, pinching, bossae and tip asymmetry are accompanying changes. Augmentations of the inadequate skeletal support can stabilize the external valve and ameliorate nasal obstruction. Traditionally, it is accomplished by alar batten grafts. The size and precise placement of this augmentation are dependent upon the corrections needed to be performed.

Loss of tip support. One of the most common iatrogenic complications of rhinoplasty is loss of tip support secondary to interrupted major or minor tip support mechanisms. Major support mechanisms include the interlocking attachment of ULC and LLC, LLC size, shape and length, and the medial crural foot plate attachments to caudal septum. Minor support mechanisms are the cartilaginous and membranous septum, the interdomal ligament (fibrous connective tissue attachment between the medial and middle crura) and LLC attachment to the skin.

Recognition of the effects of incisions and resections during rhinoplasty that violate these support mechanisms should be thoroughly appreciated. The exact causes of loss of support should be identified and countered. Commonly, cartilage grafts are used for augmentation to establish acceptable contours: alar batten grafts are used to support the alar rims, strut grafts stabilize the medial crura, and tip grafts support and contour the tip. Once identified, loss of tip support can be overcome via invasive or minimally invasive augmentation type techniques.

\section{The aging nose}

Aging is associated with changes in nasal aesthetics. These include downward rotation of the tip lobule, creating an acute columellar lobule angle, maxillary alveolar hypoplasia with 
resultant divergence of the medial crural feet and columellar shortening. The aesthetic result is a relatively longer nasal length, a droopy tip appearance and an apparently prominent dorsal hump (4).

\section{Material and methods}

\subsection{Pretreatment considerations}

Achieving satisfactory aesthetic outcomes relies on realistic expectations of properly selected patients in conjunction with precise execution of a selected technique that optimally addresses the deformity. During the initial interview, the patient's desires and goals should be carefully assessed in terms of realistic expectations of what can be accomplished. Medical history should be reviewed with focus on use of medication or supplements that might increase bleeding (e.g., nonsteroidal anti-inflammatory drugs, salicylate drugs, vitamin E, Omega-3, Ginseng, Ginko Biloba), allergies, history of cold sores, previous nasal operations or dermal filler treatments, and whether the patient is pregnant or lactating.

Before injection, the patient is counseled about the potential benefits against the inherent limitations of minimally invasive rhinosculpturing. Treatment course, possible adverse events, and the anticipated durability of treatment are reviewed. Following a full disclosure, informed consent is obtained and pretreatment photographs are taken in frontal, oblique, lateral and basal views.

\subsection{Radiesse - the preferred injectable filler}

Radiesse (Bioform Medical Inc, San Mateo, California) is an injectable filler material composed of synthetic CaHA microspheres $(30 \%)$, suspended in an aqueous carrier gel $(70 \%)$. CaHA is the primary constituent of bone and teeth and has been in use for more than 20 years in medicine $(11,12)$.

Injectable CaHA is FDA approved for correction of moderate to severe facial wrinkles and folds and for restoration/correction of lipoatrophy in patients with HIV (13).

Following injection, the carrier gel is gradually absorbed and CaHA particles remain. Local histolocytic and fibroblastic response at the injection site result in neocollagenesis around the microspheres (14), thus contributing to the prolonged effects. Results achieved following injection remain almost unchanged until 12 months. After 12-18 months, the achieved volumes begin to diminish, though some results can be noted 24 months post-injection. The average longevity therefore could be considered to be 12-18 months (15-19). Longer duration of effect, up to 24 months, can be seen in some patients with a touch-up at one year of half the amount injected the first time (20).

$\mathrm{CaHA}$ was reported to generate longer lasting results and higher levels of patient satisfaction in 2 head to head studies with widely used HA fillers $(21,22)$. Due to its composition and inherent biocompatibility, Radiesse is particularly suitable injectable material for augmentation of bony and cartilaginous contour deformities in the nasal area.

\subsection{Pre-injection procedure}

Before treatment, injection sites should be carefully marked by a washable marker with the patient in an upright position, and prepared with suitable antiseptic. Topical anesthesia using EMLA (lidocaine 2.5\% and prilocane 2.5\%) is applied for at least 15-20 minutes before 
injection. As regional infiltrative anesthesia frequently distorts surface landmarks, my personal preference is to premix 1cc of Radiesse with $0.3 \%$ lidocaine, a ratio equivalent to the widely used FDA-approved HA fillers. Radiesse is supplied in $1.5 \mathrm{cc}$ or $0.8 \mathrm{cc}$ disposable syringes with Leur-lock fittings and should be injected with a 27-gauge 0.25 inch $(6 \mathrm{~mm})$ long needle.

\subsection{Injection techniques}

Radiesse should ordinarily be injected into the subdermal supraperiosteal plane in a retrograde fashion using a linear threading, fanning or cross-hatching technique, depending on the area being treated. The needle is placed at an angle of 30-45 degrees to the skin, and a subcutaneous space or track is created. Needle location is assessed with the index finger of the non-dominant hand to avoid visible nodules that might be created by superficial injection. Slow, continuous, even pressure applied to the syringe plunger whilst withdrawing the needle assures adequate control and helps to avoid product palpability. Frequent reassessment throughout the procedure is recommended when the patient is in the upright position, as the product is deposited incrementally. Overcorrection is to be avoided. As a practical rule, it is advisable to gradually achieve the final result over 2-3 sessions.

Systematic approach in rhinosculpturing is recommended starting with the nasal root and dorsum and proceeding to the supratip area, tip lobule and finally, the nasal base. Each injected area should address a specific deformity or aesthetic imperfection outlined during nasal examination and nasal aesthetic analysis. Important technical considerations and treatment goals will be discussed in relation to the above-mentioned anatomical regions:

\section{Nasal root}

Radiesse is injected into the supraperiosteal plane in the intended area using linear threading technique, starting in the midline. The index and thumb of the nondominant hand should be placed on either side of the nasal bones to prevent product migration. By doing so, the nasofrontal angle is increased (displaced cephalically), thus improving radix location in cases of low radix and the apparent short nose.

\section{Nasal dorsum}

Careful inspection of the light reflex from an upright standing position can reveal any irregularities in dorsal contour. Proper material deposition addresses dorsal contour irregularities, low radix, inverted V deformity, and improves the dorsal concavity of saddle nose deformity.

\section{Nasal tip}

The tip of the nose area is sensitive, so the injection point should be properly planned. It is recommended to start in the midline, slightly caudal to and between the tip-defining points, so a larger surface area can be approached using a single needle puncture. Injection depth is in the subcutaneous plane above the LLC. The index and thumb of the nondominant hand pinch the membranous caudal septum in order to alleviate pain and to stabilize the injected area. Next, the needle is advanced up to the supratip break point. (This point defines the cephalic limit of the nasal tip and is created by the 
difference between the projection of the tip-defining point and the height of the nasal dorsum.)

Injection while the needle is withdrawn can effectively correct a bifid tip and enhance tip projection. When continuing injection using the fanning technique, simulating an isosceles triangle, whose shanks point toward the medial portion of the lateral crura, pinched tip is improved and tip support is stabilized. This is especially the case when loss of tip support is due to excessive excision of the medial half of the lateral crus. By augmenting the inadequate skeletal support of the external valve, external valve incompetence can be improved.

\section{Nasal base}

Tip rotation, projection and support can be modified by augmentation. Injection of Radiesse into the central nasal base above the anterior nasal spine of the maxillary bone advances the premaxilla and opens the nasolabial angle, thus rotating the tip cephalically and improving a droopy tip. The needle is inserted at $45^{\circ}$ to the upper lip toward the nasal spine and Radiesse is deposited $1 \mathrm{~mm}$ supraperiosteally while the index finger and thumb of the nondominant hand grasp the membranous septum cephalically. If further enhancement of tip support, projection and rotation is desired, augmentation material is placed between the medial crura toward the tip lobule, simulating columellar strut. By doing so, columellar show improves.

\section{Functional nasal problems}

Common causes of airway obstructions are internal and external valvular incompetence and inadvertent loss of tip support. Airway obstruction and loss of skeletal support require augmentation type procedures.

\section{Spreader graft injection}

With the aid of a head light, a double-hook or a nasal speculum is placed onto the nostrils. The alar rims are averted with the nondominant hand, thus exposing the internal nasal valve. Radiesse is injected into the submucoperichondrial and submucosal planes at the interface between the upper lateral cartilages and the dorsal cartilaginous septum using a 25 -gauge 1.25 inch needle, in a retrograde linear threading technique. The injection serves as a spacer and opens the anterior septal angle, thereby increasing the cross-sectional area of the internal valve. This maneuver significantly increases the airflow into the nasal passages.

\section{Alar batten graft injection}

External valve incompetence requires structural support provided traditionally by placement of alar batten grafts. Once the site of collapse is identified during inspiration, the area is marked. The needle is threaded through the vestibule toward the supra-alar crease at the junction of the ULC and LLC, and injection is performed in a fanning technique. Alternatively, needle puncture is performed percutaneously toward the premarked area. Depth of injection reaches the subdermal and supra-perichondrial plane. Injection of alar batten reinforces the ala and nasal sidewall that had been prone to collapse with respiration. It should be noted that alar batten injection is difficult technically due to the tight skin envelope. Small doses, deposited over 2-3 sessions in an incremental fashion is recommended. 


\subsection{Post-injection consideration}

Following injection, immediate molding and massage is advisable to smooth the surface and avoid irregularities. Injection site reactions such as redness and swelling are frequent and resolve spontaneously. Application of ice on to the injected areas helps to reduce tissue edema and ecchymosis. Patients are instructed to avoid the sun, tanning lights, sauna and intense facial treatment for at least 24 hours. Patient follow-up visits are typically scheduled 2 weeks post-injection. Micropore-like tapes are applied to the injected areas to diminish swelling. Refinement treatments are provided as needed.

\section{Case presentations}

\subsection{Case presentation 1}

Figure 4a. A 40-year-old female presented with inadequate tip projection and superior tip rotation ("porcine deformity") following rhinoplasty. A step-off deformity in the right nasal sidewall is presented secondary to overresection of the bony and cartilaginous roof. The lateral crus is deficient as well. The remaining cartilaginous rim appears knuckled owing to contractural healing forces acting on weakened cartilages (bossae) in a patient with thin skin. Physical examination revealed incompetence of the internal and external valves.

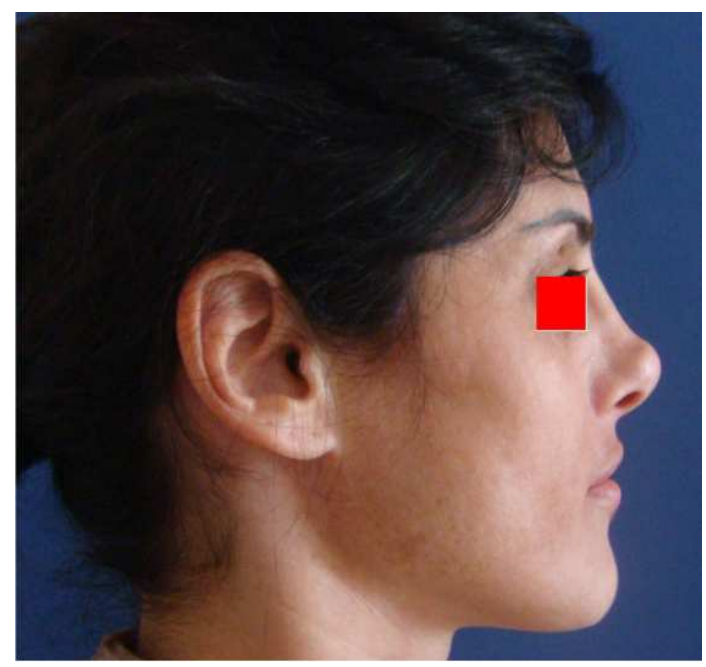

Fig. 4a. Preinjection lateral view demonstrating an inadequate tip projection, superior tip rotation, and step-off deformity in the right nasal side wall and bossae following prior rhinoplasty

Figure 4b. Post-injection photograph following spreader graft injection, and alar batten graft injection to support the internal and external valves, respectively. Radiesse ${ }^{\mathrm{TM}}$ was also injected to the nasal sidewall to smooth the step-off deformity and to the caudal dorsum at the supratip region to de-emphasize the overrotated tip (nasolabial angle was $104^{\circ}$ before treatment and $92^{\circ}$ following injection). Support of the skeletal deficient areas improved both form and function. The end result is an aesthetically pleasing nasal profile and well supported and projected tip. 


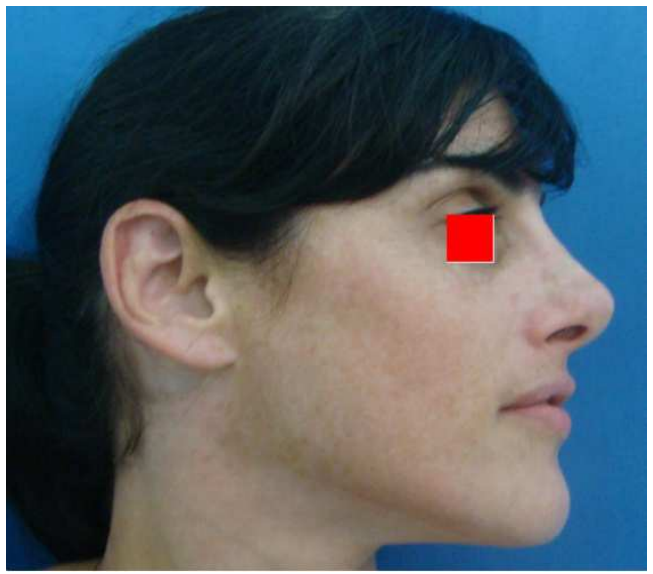

Fig. 4b. Postinjection lateral view showing improved nasal profile and well supported and projected tip

\subsection{Case presentation 2}

Figure 5a. Frontal view of a 47-year-old man with a history of 2 previous rhinoplastic surgeries and cartilage grafts (septal, conchal) placement during his revision rhinoplasty 5 years prior to presentation. Nasal examination revealed a deviated nasal bony base to right upper third, irregular nasal dorsum with slight saddle nose deformity, collapse of the left middle vault, collapse of both alar wings (left side greater than the right side), inadequate tip projection, asymmetric tip with deviation to the right, right side alar retraction and slightly retracted columella.

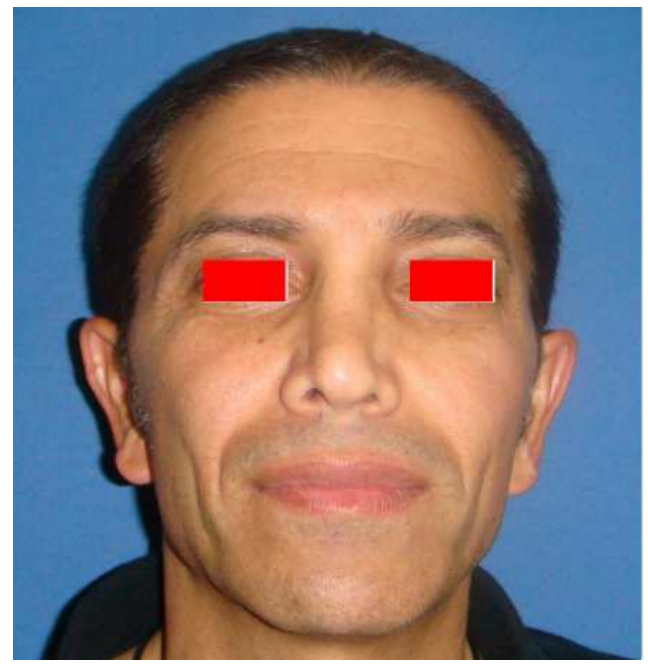

Fig. 5a. Preinjection frontal view demonstrating a deviated nasal bony base to the right, irregular nasal dorsum with slight saddle nose deformity, collapse of the left middle vault and alar wings and an asymmetric, inadequately projecting tip 
Figure 5b. Frontal view following minimally invasive rhinosculpturing. Nasal sidewalls and dorsal augmentations were performed along with spreader grafts and alar batten graft injections. The unsightly right side bony irregularity was eliminated, the saddle deformity was corrected and dorsal contour was improved. Tip support was stabilized and symmetry was improved. Alar collapse was eliminated on the right side and improved on the left side (left alar collapse was eliminated in a subsequent session.) Spreader graft and alar batten graft injections enabled improved airflow due to the corresponding increase in the internal nasal valve angle and external valve area, respectively.

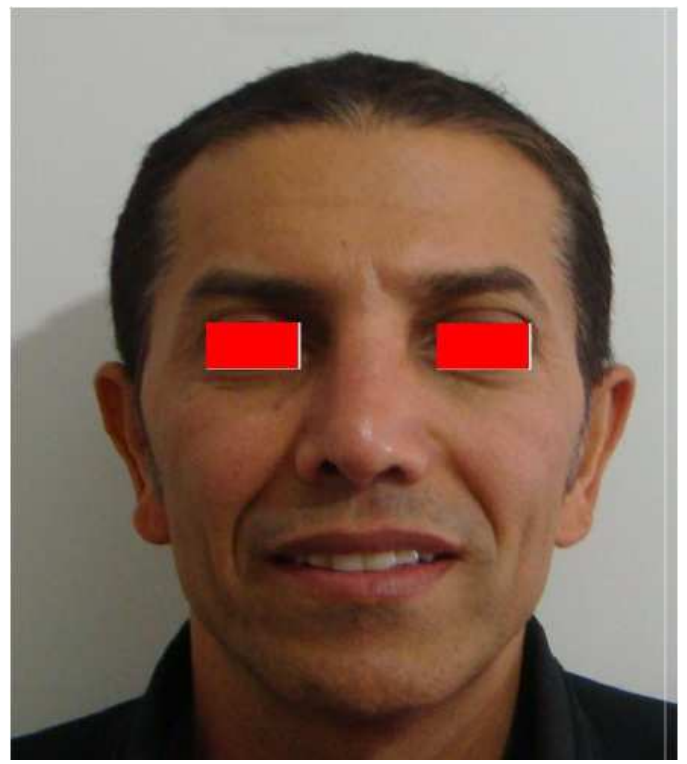

Fig. 5b. Frontal view following minimally invasive rhinosculpturing. Note the improved dorsal contour, enhanced nasal symmetry and smoothened skeletal irregularities

\section{Discussion}

During the last decade, minimally invasive rejuvenation procedures have gained widespread popularity and have become an indispensible component of today's modern cosmetic procedures. The diversity/blossoming of today's product options, recent innovations in injectable filler technology, and availability of fillers with documented safety profile, acceptable longevity, biocompatibility and low adverse problems pave the path to this paradigm shift. Moreover, minimally invasive facial procedures offer minimal downtime, less pain, no scars and a lower cost alternative compared to invasive procedures.

Rhinosculpturing using minimally invasive technique provides an attractive alternative to conventional rhinoplasty in selected cases. These include virgin noses necessitating augmentation type procedure for aesthetic refinement, correction of post-rhinoplasty contour defects, treating the aging and ethnic nose, and ameliorating/reversing selected functional nasal impairments. Nevertheless, conventional rhinoplasty remains a pre- 
eminent treatment in cases necessitating reduction type interventions, septal deformities, and nasal deformities otherwise not amenable to minimally invasive techniques.

As with any aesthetic procedure, the aesthetic outcome is the ultimate measure of proper selection and execution of a technique that is optimally tailored to the deformity addressed.

Inadequate nasal skeletal structure and support mandates the need for implant materials to improve both form and function. Augmentation materials can be broadly categorized as autografts, homografts, and alloplasts. An ideal augmentation material should approximate closely the shape, consistency and strength of the deficient nasal framework. It should be easily obtainable, capable of being shaped, biocompatible, resistant to extrusion or resorption by the body, and cost-effective. Myriad materials have been used over the years, each with its inherent advantages and liabilities (23).

Autologous cartilage grafts have become widely used as an effective autogenous supportive tissue. Sources include septal conchal and costal cartilage. If available, septal cartilage remains the cartilage of choice because of its rigidity, proximity, and straight contour. Septal grafts are commonly used as dorsal/radix grafts, spreader grafts, tip grafts, columellar struts and lateral crural and alar batten grafts. Despite their unsurpassed biocompatibility, disadvantages of cartilage grafts include limited supply, potential donor site morbidity, poorly predictable resorption rates and potential palpability, visibility or warping.

Injectable materials allow for non-invasive nasal augmentation and sculpture. Ideally, they should be non-toxic, non-allergic, biocompatible, easy to use, long lasting (yet, nonpermanent), inexpensive and reversible. They should demonstrate a high safety profile and produce a predictable result with minimal downtime.

Radiesse is a semi-solid, cohesive, completely biodegradable, deep subdermal implant. Its principle component is synthetic CaHA, the primary mineral constituent of bone and teeth. Results from in vivo and in vitro safety studies demonstrate that injectable CaHA is biocompatible, non-toxic, non-irritating and non-antigenic (24). Clinical, histologic and electron microscopic findings after in vivo injection have demonstrated dermal matrix integration in biopsy samples harvested from human volunteers (12). This CaHA injection provides scaffolding for collagen growth, thereby prolonging the duration of the effect. These histologic findings were accompanied by evidence of maintained clinical improvement (12). Biocompatibility studies on CaHA implants have all been characterized by minimal, if any, inflammatory response with no foreign body reaction or evidence of local systemic toxicity $(11,12)$. Furthermore, there was neither ossification nor migration from the injection sites (20).

Two multicenter randomized trials comparing CaHA injections with the widely used HA fillers demonstrate that $\mathrm{CaHA}$ injections generate longer lasting results and higher levels of patient satisfaction $(21,22)$. This might be explained by Radiesse's unique composition and by the higher elasticity and viscosity of Radiesse compared to the leading HA based dermal fillers (25).

Radiesse's chemical composition, physical properties and documented high safety profile render it valuable as the preferred injectable filler for augmentation type rhinosculpturing. Minimally invasive rhinosculpturing using Radiesse offers an attractive alternative to conventional rhinoplasty for patients who are actively hoping to avoid invasive surgery, and for those who are not candidates for surgery because of serious comorbidities. Patients 
wishing to appreciate the benefits of rhinosculpturing before a subsequent surgery and those who refuse revision surgery are likewise candidates for minimally invasive approach to rhinoplasty.

Selected functional nasal impairment can be ameliorated or even reversed via minimally invasive approach using Radiesse. Nyte (26) reported a spreader graft injection with Radiesse for a non-surgical solution in internal valve collapse in 23 patients. Alar batten graft injection with Radiesse can be used as a minimally invasive approach for external valve collapse. Radiesse might well serve as a viable alternative for other cartilage grafts such as dorsal cartilage graft, radix graft, tip grafts and columellar strut to contour structural nasal deficiencies. Cartilage graft injection using Radiesse spares donor sites, has minimal downtime, relatively longer term longevity, and is efficacious and cost-effective. Cartilage graft injection has low risk of extrusion, palpability or visibility.

Rhinosculpturing via minimally invasive approach should be considered as an alternative in cases mandating revision rhinoplasty where surgical expertise is of utmost importance. In those cases minimally invasive rhinosculpturing might spare the inevitable inherent difficulties posed on rhinoplasty in a previously operated nose.

In conclusion, Radiesse ${ }^{\mathrm{TM}}$ rhinosculpturing adds to the armamentarium of the rhinoplasty surgeon. It is an easy to perform procedure that might spare an operation and provides high patient satisfaction. Although much can be achieved by minimally invasive approach to rhinosculpturing, one must be cognizant of the fact that when selecting alternatives to address specific deformities, proper patient selection, detailed systematic analysis of the anatomical variables, artistic perception and precise execution are of paramount importance.

\section{References}

[1] Oneal RM, Beil RJ Jr, Schlensinger J. Surgical anatomy of the nose. Clin Plast Surg 1996;23(2):195-222.

[2] Powell N, Humphrey B. Proportions of the aesthetic face. New York: Thieme-Stratton; 1984.

[3] Tardy ME, Becker DG, Weingerg MS. Illusion in rhinoplasty. Facial Plast Surg 1995;11:117-138.

[4] Rohrich RJ, Hollier JR LH, Janis JE, Kim J. Rhinoplasty with advancing age. Plast Reconstr Surg 2003;114(7):1936-1944.

[5] Larrabee WFJr. Facial beauty. myth or reality. Arch Otolaryngol Head Neck Surg 1997;123:571-2

[6] American Society for Aesthetic Plastic Surgery. 1997-2010 Cosmetic Surgery National Data Bank Statistics. http://www.surgery.org. Accessed October,10 2010.

[7] Petroff MA, McCollough EG, Hom D, Anderson JR. Nasal tip projection: quantitative changes following rhinoplasty. Arch Otolaryngol Head Neck Surg 1991;117:783-8.

[8] Ricketts RM. Divine proportion in facial esthetics. Clin Plast Surg 1982:9:401-22.

[9] Constantian MB. Practical nasal aesthetics. In: Habal M, ed. Advances in Plastic and Reconstructive Surgery. St Louis, Mosby-Year Book, 1991:85-107.

[10] Becker DG, Becker SS. Reducing complications in rhinoplasty. Otolaryngol Clin North Am 2006;39:475-92. 
[11] Havlik RJ; PSEF DATA Committee. Hydroxylapatite. Plast Reconstr Surg 2002;15:11769.

[12] Hobar PC, Pantaloni M, Byrd MS. Porous hydroxyapatite granules for alloplastic enhancement of the facial region. Clin Plast Surg 2000;27:557-69.

[13] Radiesse [package insert], San Mateo, CA: Bioform Medical Inc., 2009.

[14] Marmur ES, Phelps R, Goldberg D, Marmur et al. (2004) Clinical, histologic and electron microscopic findings after injection of a calcium hydroxylapatite filler. J Cosmet Laser Ther. 2004 Dec;6(4):223-6.

[15] Tzikas TL. Evaluation of Radiesse FN: A new soft tissue filler. Dermatol Surg 2004;30:764-8.

[16] Kanchwala SL, Holloway L, Bucky LP. Reliable soft tissue augmentation: a clinical comparison of injectable soft-tissue fillers for facial-volume augmentation. Ann Plast Surg 2005;55:30-5.

[17] Jacovella PF. Calcium hydroxylapatite facial filler (Radiesse): indications, technique, and results. Clin Plast Surg 2006;33:511-23.

[18] Jansen DA, Graivier MH. Evaluation of calcium hydroxylapatite-based implant (Radiesse) for facial soft tissue augmentation. Plast Reconstr Surg 2006 Sep;118(3 Suppl):22S-30S, discussion 31S-33S.

[19] Silvers SL, Eviatar JA, Echavez MI, Pappas AL. Prospective, open-label, 18-month trial of calcium hydroxylapatite (Radiesse) for facial soft-tissue augmentation in patients with human immunodeficiency virus-associated lipoatrophy: one-year durability. Plast Reconstr Surg 2006 Sep;118(3 Suppl):34S-45S.

[20] Feldmerman LI. Radiesse for facial rejuvenation. Cosmet Dermatol 2005;18:823-6.

[21] Moers Carpi MM, Tufet JO. Calcium hydroxylapatite versus nonanimal stabilized hyaluronic acid for the correction of nasolabial folds: a 12-month, multicenter, prospective, randomized, controlled, split-face trial. Dermatol Surg 2008 Feb;34(2):210-5.

[22] Moers-Carpi M, Vogt S, Santos BM, Planas J, Vallve SR, Howell DJ. A multicenter, randomized trial comparing calcium hydroxylapatite to two hyaluronic acids for treatment of nasolabial folds. Dermatol Surg. 2007;33 Suppl 2:S144-51.

[23] Lovice DB, Mingrone MD, Toriumi DH. Grafts and implants in rhinoplasty and nasal reconstruction. Otolaryngol Clin North Am 1999;32(1):113-41.

[24] Hubbard WH. Bioform implants. Biocompatibility. Franksville, Wis: Bioform, Inc., 2003.

[25] BioForm Medical Inc., 2009, data on file.

[26] Nyte CP. Spreader graft injection with calcium hydroxylapatite: a nonsurgical technique for internal valve collapse. Laryngoscope 2006:116:1291-2 


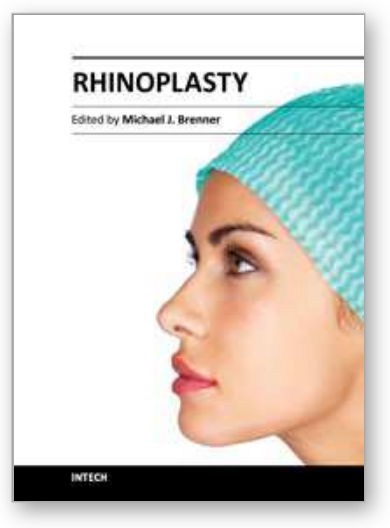

\author{
Rhinoplasty \\ Edited by Dr. Michael Brenner
}

ISBN 978-953-307-849-6

Hard cover, 146 pages

Publisher InTech

Published online 09, December, 2011

Published in print edition December, 2011

Rhinoplasty is one of the defining procedures of plastic and reconstructive surgery. Its roots stem from early efforts in nasal reconstruction to the emergence of modern rhinoplasty. This book describes the latest clinical and research perspectives in rhinoplasty and balances structural correction with aesthetic refinement. With treatises on rhinoplasty from a diverse set of thought leaders from around the world, the collective experience of this books' authors cover cosmetic and reconstructive approaches with a wealth of proven and innovative approaches ranging from minor refinement to major reconstruction. This diversity reflects the inherent complexity of the art and science of rhinoplasty. Discussion of structural approaches is balanced by consideration of judicious resection and refinement. The overarching goal is to instill an understanding of the subtleties of nasal structure and how the natural complexities of nasal anatomy can be adapted to maximize both function and natural appearance.

\title{
How to reference
}

In order to correctly reference this scholarly work, feel free to copy and paste the following:

S. Cohen (2011). Minimally Invasive Approach for Rhinoplasty, Rhinoplasty, Dr. Michael Brenner (Ed.), ISBN: 978-953-307-849-6, InTech, Available from: http://www.intechopen.com/books/rhinoplasty/minimally-invasiveapproach-for-rhinoplasty

\section{INTECH}

open science | open minds

\author{
InTech Europe \\ University Campus STeP Ri \\ Slavka Krautzeka 83/A \\ 51000 Rijeka, Croatia \\ Phone: +385 (51) 770447 \\ Fax: +385 (51) 686166 \\ www.intechopen.com
}

\author{
InTech China \\ Unit 405, Office Block, Hotel Equatorial Shanghai \\ No.65, Yan An Road (West), Shanghai, 200040, China \\ 中国上海市延安西路65号上海国际贵都大饭店办公楼405单元 \\ Phone: +86-21-62489820 \\ Fax: $+86-21-62489821$
}


(C) 2011 The Author(s). Licensee IntechOpen. This is an open access article distributed under the terms of the Creative Commons Attribution 3.0 License, which permits unrestricted use, distribution, and reproduction in any medium, provided the original work is properly cited. 